\title{
Paul Bert als Politiker, Pädagog und Begründer der Höhenphysiologie
}

\author{
Von Nikolaus Mani, Madison (Wis., USA)
}

Paul Bert lebte von 1833 bis 1886. Er starb im Alter von 53 Jahren als erster Generalresident der Französischen Republik in Indochina.

Berts Name ist mit der frühen Geschichte der Dritten Republik aufs engste verbunden. Er war glühender Republikaner, Kampfgenosse des Erzrepublikaners und Volkstribunen LÉon GambetTa, Abgeordneter in der französischen Nationalversammlung, während kurzer Zeit Erziehungsminister, Publizist, redegewaltiger Antiklerikaler, erklärter Gegner der Jesuiten, Pädagoge, treibende Kraft der französischen Schulreform in den achtziger Jahren. Als Physiologe war er Schüler und Nachfolger Claude Bernards.

Paul Bert wurde in Auxerre (Departement Yonne) geboren. Eine sanfte, verständnisvolle Mutter und ein tatkräftiger, herrschgewohnter Vater überwachten die Erziehung des jungen Paul. Als Zwanzigjähriger zog er nach Paris. Hier studierte er ohne rechte Begeisterung, dem Vater gehorchend, schlecht und recht Jurisprudenz.

Der erste Forscher, der Bert tief beeindruckte, war der vergleichende Anatome Louis Pierre Gratiolet. Wie Bert später scherzhaft bemerkte, wurde er von Gratiolet geradezu zur Wissenschaft verführt (ce séducteur à la science). Eindringlich schilderte Bert, wie Gratiolet mit flammenden Worten die Deszendenztheorie verdammte und die Dignität und Singularität des Menschen in der Schöpfung pries. Bert selbst wurde durch Darwins Buch Über die Entstehung der Arten im Innersten aufgewühlt (une révolution quasi maladive).

Bert studierte Medizin und Naturwissenschaften. Entscheidend wurden die Jahre 1863 bis 1866. In dieser Zeit arbeitete Bert als «préparateur» Cl. Bernards am Collège de France. Hier empfing er sein methodisches und gedankliches Rüstzeug. Hier erfuhr er, was eine einfallsreiche Methodik, eine strenge experimentelle Kritik und eine luzide Interpretation physiologischer Fakten zu leisten vermochte. Im Jahre 1869 wurde Bert Nachfolger Cl. Bernards auf dem physiologischen Lehrstuhl der Sorbonne.

Dann kam der Deutsch-Französische Krieg und mit ihm die große Zäsur in Berts Leben. Die französische Armee, nach den Worten des Kriegsministers angeblich bis zum letzten Gamaschenknopf gerüstet, kapitulierte bei 
Sedan. Napoleon III., an Körper und Geist gebrochen, verschwand von der politischen Bühne. In Paris bildete sich inmitten unbeschreiblicher Verwirrung eine Regierung der nationalen Verteidigung.

Ein Stimmungsbild jener Tage: Cl. Bernard schrieb am Ende seiner Senatorenlaufbahn an seine Freundin $M^{\text {me }}$ Raffalovic: «Le délire commence, Où sommes-nous, où allons-nous, et que deviendrons-nous ? Pauvre France.»

Gambetta rief zu neuem Kampfe auf. Er entwich mit einem Ballon in die Provinz und rief zum äußersten Widerstande auf. P. Bert schloß sich ihm an. Zur größten Enttäuschung seines Lehrers Cl. Bernard organisiert er als Präfekt des Nordens eine aussichtslose Verteidigung. Bert geißelte jede defaitistische Regung und wollte nicht kapitulieren. Bernard hielt ihm später mit schneidender Schärfe entgegen: «Mein armer Bert, seitdem Sie Politiker geworden sind, haben Sie aufgehört ein Mann der Wissenschaft zu sein.»

Nach dem Kriege blieb Bert Politiker, ohne indessen seine wissenschaftliche Tätigkeit und seinen Lehrstuhl an der Sorbonne aufzugeben. Er führte ein Doppelleben: Die Existenz des Politikers in der fiebrigen Atmosphäre der jungen Dritten Republik wechselte ab mit der Tätigkeit des physiologischen Forschers. In der Provinz bestritt er Wahlversammlungen, in der Hauptstadt nahm er an tumultuösen Sitzungen der Nationalversammlung teil. Er präsidierte Kommissionen. Er schrieb politische, auch populärwissenschaftliche Aufsätze, er sprach vor verschiedensten Gremien, vor Fachgenossen, aber auch vor Fabrikarbeitern. Abgehetzt eilte er ins Laboratorium, ersann neue Experimente, besprach sich mit seinen Mitarbeitern.

Für Bert sind Politik und Naturwissenschaften keineswegs gesonderte Bezirke. Die Politik muß mit wissenschaftlichem Geiste durchdrungen werden. Die Naturwissenschaften haben den menschlichen Geist befreit, sie brennen darauf, auch die menschliche Gesellschaft zu regulieren. Allzu lange ruhte die menschliche Gesellschaft auf den zwei Pfeilern des Glaubens und der Resignation. Die Wissenschaften aber sind imstande, den Glauben durch den wissenschaftlichen Beweis und die Resignation durch mutigen Kampf für wissenschaftliche Wahrheit zu ersetzen.

Damit dies möglich werde, ist eine radikale Reform des Unterrichtswesens auf allen Stufen notwendig. Vor allem auch die Reform der Elementarschulen. Bert forderte eine unentgeltliche, obligatorische und religiös neutrale Volksschule (l'obligation, la gratuité, la laïcité).

Der Priester hat aus der Volksschule zu verschwinden. Sein Bezirk ist die Kirche. In der Schule hat der «instituteur » (Volksschullehrer) das Wort, er ist der Soldat der laizistischen Republik. 
Schon auf der Stufe der Volksschule, so forderte Bert, müssen neben der Muttersprache und Rechnen naturwissenschaftliche Fächer gelehrt werden. Die Naturwissenschaften schärfen den Verstand und entwickeln das kausale Denken. Wenn der Schüler die ehernen Naturgesetze erkennt, ist er vor Aber- und Wunderglauben, aber auch vor politischer Verführung gefeit. Wer die Gesetze der Natur respektiert, wird auch die Satzungen der Republik achten (de la loi naturelle à la loi sociale, ce sentiment se conservera).

Der Unterricht in Geschichte und Paläontologie z. B. wird dem Kinde die wunderbare Entwicklung des Menschen zeigen, der Aufstieg vom Höhlenbewohner zum Kulturmenschen, vom Leibeigenen zum freien Republikaner.

Neben den Naturwissenschaften darf aber auch die schöne Literatur nicht zu kurz kommen. Die Naturwissenschaften entwickeln das logische Denken, die schönen Künste allein aber sprechen zu Herz und Gemüt.

Nach dem siebziger Krieg vertiefte sich Bert mit unerhörter Kraft und genialem Schwung in das Studium der physiologischen Wirkungen des Luftdruckes. 1878 erschien das abschließende, monumentale, bis ins feinste Detail ausgearbeitete Werk La pression barométrique.

Dieses Werk hat verschiedene Wurzeln:

1. Die früheren Studien Berts über die vergleichende Atmungsphysiologie lieferten ihm die notwendige methodische Grundlage.

2. Der in Mexiko praktizierende französische Arzt Denis Jourdanet hatte die Physiologie, vor allem aber auch die Pathologie und Klinik des Höhenaufenthaltes eingehend studiert. Er war damals der beste Kenner der Höhenmedizin. Jourdanet vermißte zuverlässige experimentelle Daten. Deshalb trat er mit Bert in Verbindung. Jourdanet bezahlte all die kostspieligen Apparate, die Bert ersann, konstruieren ließ und im physiologischen Laboratorium der Sorbonne aufstellte. Das offizielle Frankreich hat wenig Anteil an Berts Leistung.

3. Im technisch-apparativen Bereich hatten übrigens die Kliniker den Physiologen vorgearbeitet. Aerotherapie war große Mode. Forlanini in Italien betrieb sie in der komfortablen, gepolsterten Druckkabine, und Jourdanet hatte metallische Zylinder konstruiert, die eine Behandlung mit hohen und niederen Drucken erlaubten.

4. Bert war auch vom sozialen Aspekt des barometrischen Problems fasziniert: In Südamerika und Ostasien lebten ganze Völker in großen Höhenlagen. Wenn die physiologischen Bedingungen des Höhenaufenthaltes geklärt waren, ergaben sich große therapeutische und prophylaktische Möglichkeiten. 
5. Eine weitere Triebfeder war auch das ausgeprägte nationale und kulturmissionarische Bewußtsein Berts. Frankreich war die Wiege der Luftfahrt. Franzosen waren die ersten Aeronauten gewesen. Gambetta, der Freund Berts, war durch die Härte des Krieges zum ersten fliegenden Staatsmann geworden. Ihm, P. Bert, fiel die Aufgabe zu, die führende deutsche Physiologie auf diesem Teilgebiet zu überrunden.

Was fand Bert vor, als er die Arbeit begann? Eine Riesenliteratur, eine Fülle von Hypothesen, ein Spektrum an Spekulationen.

Die Bergkrankheit (mal de montagne) hatte man auf alle nur erdenklichen Ursachen zurückgeführt: Luftverdünnung, Evaporation des Blutes, Kälte, Strahlenwirkung, Kohlensäurevergiftung, Ermüdung und vieles andere mehr. In diesem spekulativen Gestrüpp gab es aber einige Lichtpunkte: Der Zürcher Arzt Konrad Meyer-Ahrens hatte den Sauerstoffmangel der Luft als wichtigste Ursache der Bergkrankheit beschrieben, und Jourdanet hatte die glückliche Bezeichnung «anoxyhémie barométrique» geprägt, die das zentrale Problem der Höhenphysiologie erfaßte: Die Sauerstoffarmut des Blutes bei niederem Luftdruck. Bert hielt fest: Was not tut, ist eine kritische, experimentelle Überprüfung der ganzen Frage.

A. In einer ersten Reihe von Experimenten studierte Bert folgendes Problem: Welche extremen Werte des Luftdruckes sind eben noch mit dem Leben vereinbar?

I. Zunächst experimentierte er mit niedrigen Drucken. Er fand: Die Versuchstiere sterben, wenn der Partialdruck des Sauerstoffs unter einen konstanten kritischen Betrag absinkt. Ob das Tier im geschlossenen Raum atmet oder in frischer Belüftung lebt, spielt für den physiologischen Effekt keine Rolle. Was zählt, ist der Partialdruck, d.h. die absolute Menge des Sauerstoffes. (La tension de l'oxygène est tout, la pression barométrique en elle-même ne fait rien ou presque rien.)

II. Nun untersuchte Bert die physiologische Wirkung hoher Luftdrucke. Zahlreiche, vielfach abgewandelte Experimente - sie alle atmen den Geist Cl. Bernards - zeigten ihm: Bei hohen Partialdrucken des Sauerstoffes stirbt das Tier. Hunde gehen unter tonisch-klonischen Krämpfen zugrunde. Damit hatte Bert eine zweite, fundamentale Entdeckung gemacht: die Beschreibung der Sauerstoffvergiftung, die Oxydose.

Erhält der Organismus zu wenig Sauerstoff, erstickt er. Lastet auf ihm ein zu hoher Sauerstoffdruck, wird er vergiftet.

B. In einer zweiten, großen Versuchsreihe studierte Bert das Verhalten der Blutgase bei verschiedenen Drucken. Es galt nun, die innere Atmung 
zu untersuchen. Wie verhält sich das Blut, dieser Mittler zwischen der äußeren Umgebung und dem inneren Milieu, in der Tiefe des Organismus? Es zeigte sich: Sinkt der äußere Luftdruck und damit auch der Partialdruck des Sauerstoffes, so nimmt der Sauerstoffgehalt des Blutes ab. Bert stellte fest: «Die Bindung von Sauerstoff an Hämoglobin kann bei niederen Drukken teilweise aufgehoben werden und vermag sich zu lösen. Die Dissoziation ist bei einem Druckabfall von $20 \mathrm{~cm} \mathrm{Hg}$ (bei $56 \mathrm{~cm} \mathrm{Hg}$ ) deutlich, sie nimmt bei noch niedrigeren Drucken erheblich zu.» (La combinaison de l'oxygène avec l'hémoglobine est susceptible de se détruire partiellement, de se dissocier, à de faibles diminutions de pression; cette dissociation est évidente dès $20 \mathrm{~cm}$ de diminution [pression de $56 \mathrm{~cm}$ ]. Elle va en augmentant quand la dépression augmente ${ }^{1}$.)

Bei hohen äußeren Drucken ändert sich die Kurve des Blutsauerstoffes radikal. Steigt man von niederen Drucken zu einer Atmosphäre empor, so sättigt sich das Blut rasch mit Sauerstoff. Nach weiterer, auch beträchtlicher Druckerhöhung wird die Sauerstoffaufnahme gering. Bernard hielt fest: «Alles scheint darauf hinzuweisen, daß bei normalem barometrischem Druck (1 Atmosphäre) eine chemische Sättigung des Oxyhämoglobins erreicht ist; über diese hinaus empfängt das Blut nur den gemäß dem Daltonschen Gesetz im Serum gelösten Sauerstoff.» (Tout semble indiquer qu'il existe, correspondant aux environs de la pression normale, un point de saturation chimique de l'oxy-hémoglobine, et qu'au delà il ne s'ajoute plus au sang que de l'oxygène dissous dans le sérum, suivant la loi de Dalton ${ }^{2}$.)

Weiterhin fand Bert: Die arterio-venöse Sauerstoffdifferenz, d.h. der Unterschied zwischen dem Sauerstoffgehalt des arteriellen und venösen Blutes, bleibt bis auf verhältnismäßig niedere Drucke konstant, d.h. etwa 6 Volumprozente Sauerstoff. Diese arterio-venöse Differenz ist von vitaler Bedeutung. Sinkt der äußere Druck stark, so wird diese Differenz kleiner, die Gewebe erhalten weniger Sauerstoff und ersticken.

C. In einer weiteren Versuchsreihe untersuchte Bert das Verhalten des Organismus bei niederem äußerem Luftdruck. Der Tierversuch zeigte: Die Atmung wird rascher, der Puls schneller. Auffällig und frühzeitig sind die neuromuskulären und zentralen Symptome: das Reaktionsvermögen sinkt,

1 La pression barométrique, S. 648.

${ }^{2}$ La pression barométrique, S. 665. 
die Sensibilität erlischt, das Tier wird teilnahmslos, verharrt schließlich wie gelähmt, wird ohnmächtig und stirbt. Der Stoffwechsel wird langsamer, die Temperatur sinkt, die Ausscheidung des Harnstoffes verkleinert sich.

D. Die Prophylaxe und Therapie der Erscheinungen, die durch niedrigen Luftdruck hervorgerufen werden, ist denkbar einfach: Sie besteht in der Zufuhr von Sauerstoff. Ein ebenso einfaches wie überzeugendes Experiment zeigte: Bei sinkendem Luftdruck bleibt das Tier am Leben, wenn gleichzeitig Sauerstoff zugeführt wird.

Nun wagte Bert den Selbstversuch. Wohlversehen mit dem Sauerstoffbehälter stieg er in die von ihm konstruierte Unterdruckkammer. Bei Senkung des Luftdruckes auf etwa $400 \mathrm{~mm}$ Quecksilber notierte er folgende Symptome: Pulsbeschleunigung, Schwindelgefühl, Übelkeit, heftige Kopfschmerzen, intellektuelle Trägheit, Verdunkelung des Gesichtsfeldes. Alle diese Beschwerden verschwanden sogleich beim Einatmen von Sauerstoff. Als Bert zur kontinuierlichen Sauerstoffatmung überging, vermochte er sehr tiefe Drucke zu ertragen, mit anderen Worten sehr große Höhe zu simulieren. Mit Sauerstoff fühlte er sich unter den Druckbedingungen des Mt. Everest wohl.

E. Ballonfahrten. Im Jahre 1874 stiegen die beiden Aeronauten CroceSpINelli und Sivel mit dem Luftballon «Etoile polaire» auf 7300 Meter Höhe. Auf Anraten Berts nahmen sie Sauerstoff behälter mit. Der belebende Effekt der künstlichen Sauerstoffatmung war eindeutig: Ohne Sauerstoffzufuhr war Croce-Spinelli gezwungen, abzusitzen. Jede Bewegung kostete ihn unsägliche Mühe. Die Linien im Spektralapparat verschwammen. Sobald er aber einige Züge aus seinem Sauerstoffballon eingeatmet hatte, erfüllte ihn neues Leben. Er fühlte sich frisch, bewegte sich mit Leichtigkeit, und die Spektrallinien waren wiederum scharf erkennbar.

Ein Jahr später unternahmen die beiden Aeronauten zusammen mit Gaston Tissandier einen weiteren Ballonaufstieg, der sie bis auf etwa 8600 Meter Höhe führte. Croce-Spinelli und Sivel starben dabei an der Höhenkrankheit, Tissandier kam knapp mit dem Leben davon. Die Aeronauten hatten zu wenig Sauerstoff mitgeführt, und was noch schlimmer war, sie hatten vergessen, ihn kontinuierlich einzuatmen. Sie waren mit wissenschaftlichen Beobachtungen und Messungen derart beschäftigt (Luftbewegungen, Feuchtigkeit, Kohlensäuregehalt, Spektralbeobachtungen), das Fieber des Höhenrekordes hatte sie derart gepackt, daß sie alle Vorsicht vergaßen. Die Euphorie kurz vor der lähmenden Schwäche, vor dem Verlust des Bewußtseins tat das ihre. 
Tissandier wollte das Mundstück des Sauerstoffbehälters fassen, aber wie gelähmt sank ihm der Arm herab. Tissandiers Schilderung ist berühmt geworden: «Gegen 7500 Meter Höhe wird man von einer außerordentlichen Erstarrung befallen. Körper und Geist werden schrittweise, allmählich schwächer, ohne daß man dessen gewahr wird, ohne daß man es weiß. Man spürt ein inneres Lustgefühl, als ob dies eine Wirkung des überflutenden Lichtes wäre. Man wird gleichgültig, man denkt nicht mehr an die gefährliche Lage, man steigt immer höher und ist glücklich darüber. Der Höhenschwindel ist kein leeres Wort. Aber soweit ich auf Grund meiner persönlichen Eindrücke urteilen kann, erscheint das Schwindelgefühl erst im letzten Augenblick; es tritt unmittelbar vor der plötzlichen, unerwarteten und unwiderstehlichen Vernichtung auf.» (Vers 7500 mètres, l'état d'engourdissement où l'on se trouve est extraordinaire. Le corps et l'esprit s'affaiblissent peu à peu, graduellement, insensiblement, sans qu'on en ait conscience. On ne souffre en aucune façon; au contraire. On éprouve une joie intérieure, et comme un effet de ce rayonnement de lumière qui vous inonde. On devient indifférent; on ne pense plus ni à la situation périlleuse ni au danger; on monte et on est heureux de monter. Le vertige des hautes régions n'est pas un vain mot. Mais autant que je puis en juger par mes impressions personnelles, ce vertige apparaît au dernier moment; il précède immédiatement l'anéantissement, subit, inattendu, irrésistible ${ }^{3}$.)

Bert zeichnete in bewundernswerter Weise das Gesamtbild der Höhenkrankheit: Sinkt der Teildruck des Sauerstoffes, so entsteht die Höhenkrankheit des Aeronauten, die Bergkrankheit des Alpinisten, die Beschwerden des experimentell hervorgerufenen Unterdruckes.

Den Ballonaufstieg schildert Bert so: Bis zu 4000 oder 5000 Metern kompensiert der Aeronaute die Sauerstoffarmut durch rasches Atmen und schnelleren Puls: Mehr Luft, die weniger Sauerstoff enthält, strömt in seine Lungen. Mehr Blut, das aber weniger sauerstoffbeladen ist, durchpulst die Gewebe. Bei 5000, 6000 Metern machen sich die ersten Symptome der Höhenkrankheit bemerkbar. Das Nervensystem reagiert zuerst: Müdigkeit, Kopfschmerzen, Übelkeit, Verdunkelung des Gesichtsfeldes; Nachlassen der geistigen Spannkraft.

Bei weiterem Aufstieg tritt lähmende Schwäche, gefährliche Euphorie ein. Dann folgt plötzlich der Verlust des Bewußtseins, die respiratorische

${ }^{3}$ La pression barométrique, S. 1064. 
Katastrophe. Die arterio-venöse Sauerstoffdifferenz ist ungenügend geworden, und die Gewebe ersticken.

\section{Zusammenfassung}

P. Berts hauptsächliche physiologische Leistung ist sein Monumentalwerk über die physiologische Wirkung des Luftdruckes. Er erkannte in vollem Umfange die physiologische Bedeutung des Partialdruckes der Atemgase. Er beschrieb die Beziehungen zwischen äußerem Partialdruck und dem Verhalten der Blutgase. Er erkannte, daß die Bergkrankheit und die Höhenkrankheit eine Folge des niederen Partialdruckes des Sauerstoffes sind. Er führte Sauerstoffgeräte ein, um die gefährlichen Folgen des Höhenaufstieges zu vermeiden. Er studierte erstmals in der Unterdruckkammer die Bedingungen des Höhenaufstieges. Er entdeckte und beschrieb die Sauerstoffvergiftung und klärte die Ursachen und Mechanismen der Caisson-Krankheit. Sein Hauptwerk erreicht durchaus das Niveau seines Meisters Cl. Bernard und wurde 1943, mitten im Kriege, ins Englische übersetzt.

\section{Bibliographie}

Ackerknecht Erwin H., Paul Bert's Triumph, Bull. Hist. Med., Supplement 3. Essays in the History of Medicine, Henry E. Sigerist (ed.), S. 16-31, Johns Hopkins Press, Baltimore 1944. Ausgezeichnete Darstellung von Berts politischem und geistesgeschichtlichem Hintergrund mit wichtiger Bibliographie.

BÉrillon E., L'œuvre scientifique de Paul Bert, Paris 1887. Analyse der biologisch-physiologischen Leistung Paul Berts.

Bert Paul, Barometric Pressure: Researches in Experimental Physiology, translated by Mary Alice und Fred A. Hitchcock, College Book Company, Columbus (Ohio) 1943.

- La Morale des Jésuites, avec une dédicace à M. Freppel, évêque d'Angers, G. Charpentier, Paris 1880.

- La pression barométrique: Recherches de physiologie expérimentale, G. Masson, Paris 1878.

Dubreuil Leon, Paul Bert, F. Alcan, Paris 1935. Biographie mit besonderer Würdigung des politischen und zeitgenössischen Hintergrunds.

Ducloz Jean, L'enfance et la jeunesse de Paul Bert, Bull. Soc. Sci. Hist. et Nat. de l'Yonne 78, (1924) 5-102. Ansprechende Beschreibung von Berts Kindheit und Jugend.

Mumenthaler Marco, Die Erscheinungen des Sauerstoffmangels. Ein geschichtlicher Beitrag, Gesnerus 16 (1959) 47-65. 


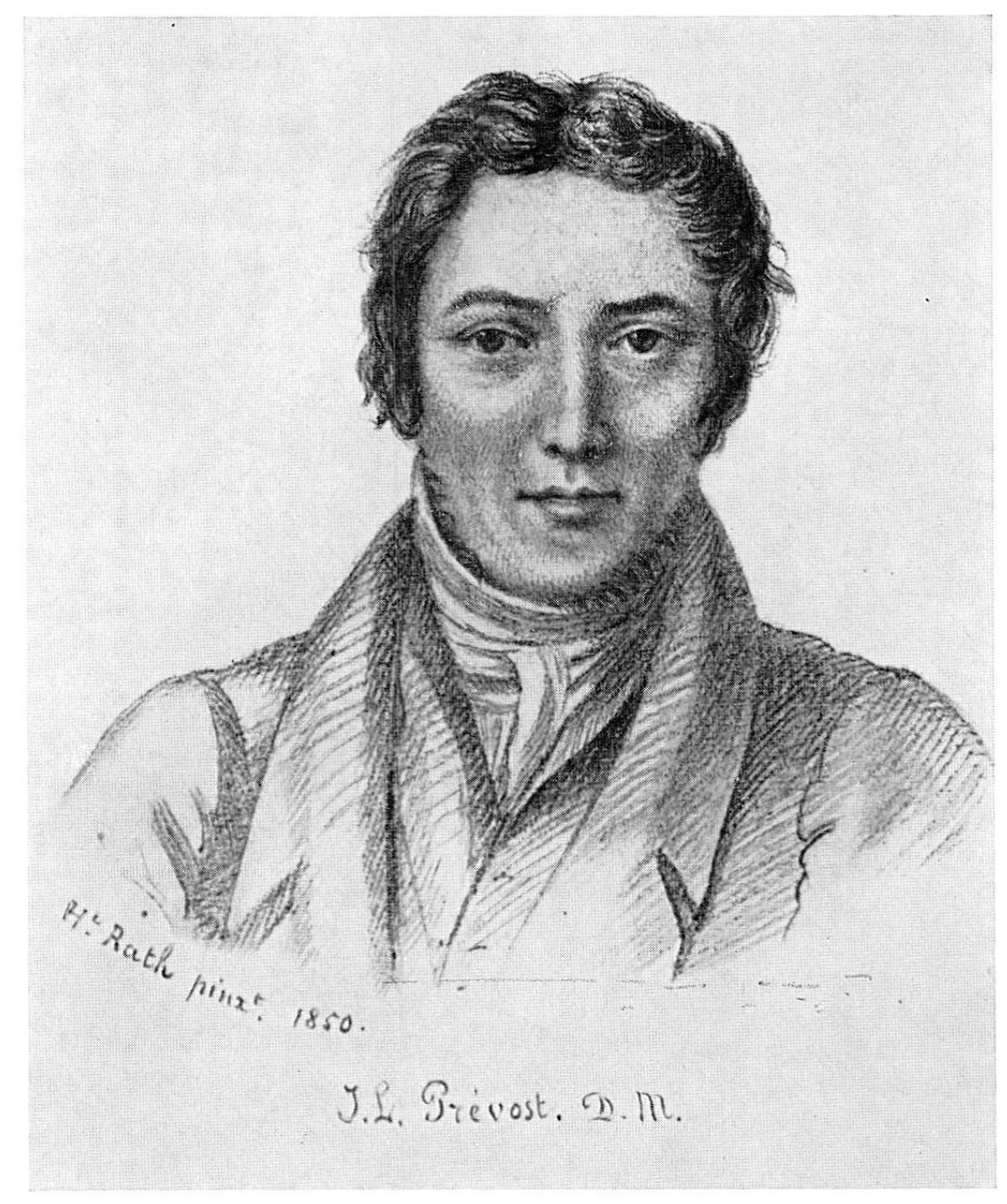

